\title{
Benefits of biphasic calcium phosphate hybrid scaffold-driven osteogenic differentiation of mesenchymal stem cells through upregulated leptin receptor expression
}

Chi-Chien Niu ${ }^{1,2^{*}+}$, Song-Shu Lin ${ }^{1+}$, Wen-Jer Chen ${ }^{1,2+}$, Shih-Jung Liư ${ }^{3}$, Lih-Huei Chen ${ }^{1,2}$, Chuen-Yung Yang ${ }^{1}$, Chao-Jan Wang ${ }^{4}$, Li-Jen Yuan ${ }^{1,2}$, Po-Han Chen ${ }^{1}$ and Hsiao-Yang Cheng ${ }^{4}$

\begin{abstract}
Background: The use of mesenchymal stem cells (MSCs) and coralline hydroxyapatite (HA) or biphasic calcium phosphate (BCP) as a bone substitute for posterolateral spinal fusion has been reported. However, the genes and molecular signals by which MSCs interact with their surrounding environment require further elucidation.

Methods: MSCs were harvested from bone grafting patients and identified by flow cytometry. A composite scaffold was developed using poly(lactide-co-glycolide) (PLGA) copolymer, coralline HA, BCP, and collagen as a carrier matrix for MSCs. The gene expression profiles of MSCs cultured in the scaffolds were measured by microarrays. The alkaline phosphatase (ALP) activity of the MSCs was assessed, and the expression of osteogenic genes and proteins was determined by quantitative polymerase chain reaction (Q-PCR) and Western blotting. Furthermore, we cultured rabbit MSCs in BCP or coralline HA hybrid scaffolds and transplanted these mixtures into rabbits for spinal fusion. We investigated the differences between BCP and coralline HA hybrid scaffolds by dual-energy X-ray absorptiometry (DEXA) and computed tomography (CT).

Results: Tested in vitro, the cells were negative for hematopoietic cell markers and positive for MSC markers. There was higher expression of 80 genes and lower of 101 genes of MSCs cultured in BCP hybrid scaffolds. Some of these genes have been shown to play a role in osteogenesis of MSCs. In addition, MSCs cultured in BCP hybrid scaffolds produced more messenger RNA (mRNA) for osteopontin, osteocalcin, Runx2, and leptin receptor (leptin-R) than those cultured in coralline HA hybrid scaffolds. Western blotting showed more Runx2 and leptin-R protein expression in $B C P$ hybrid scaffolds. For in vivo results, $3 D$ reconstructed $C T$ images showed continuous bone bridges and fusion mass incorporated with the transverse processes. Bone mineral content (BMC) values were higher in the BCP hybrid scaffold group than in the coralline HA hybrid scaffold group.

Conclusions: The BCP hybrid scaffold for osteogenesis of MSCs is better than the coralline HA hybrid scaffold by upregulating expression of leptin-R. This was consistent with in vivo data, which indicated that BCP hybrid scaffolds induced more bone formation in a spinal fusion model.
\end{abstract}

Keywords: Coralline HA, BCP, MSCs, Osteogenesis, Leptin receptor

\footnotetext{
* Correspondence: niuchien@adm.cgmh.org.tw

${ }^{\dagger}$ Equal contributors

'Department of Orthopaedics, Chang Gung Memorial Hospital, No. 5,

Fu-Hsing Street 333, Kweishan, Taoyuan, Taiwan

${ }^{2}$ College of Medicine, Chang Gung University, Taoyuan, Taiwan

Full list of author information is available at the end of the article
} 


\section{Introduction}

Autogenous bone is the most effective graft material for the repair of bone defects or for spinal fusion; however, donor site morbidity is a major limitation on its clinical use [1]. To avoid this limitation, one tissue engineering approach would be to combine cells capable of osteogenic activity with an appropriate scaffolding material to stimulate bone regeneration and repair. The use of calcium phosphate ceramics as substitutes for bone grafts is steadily increasing due to ongoing improvement of the materials. The most widely used calcium phosphate ceramics are porous hydroxyapatite (HA), phosphate ( $\beta$-TCP), and mixtures of these two that are known as biphasic calcium phosphate (BCP) ceramics [2-4].

Mesenchymal stem cells (MSCs) have the potential to differentiate into lineages of various mesenchymal tissues, including bone, cartilage, fat, tendon, and muscle. Many surface antigens are often expressed on human MSCs, such as CD146, CD106, CD105, CD90, Stro- 1 , and $\alpha$-SMA $[5,6]$. MSCs have great appeal for tissue engineering and therapeutic applications. MSCs combined with HA [7] or BCP [8] ceramics have been shown to induce bone formation. In addition, the use of MSCs on HA [9] or BCP [10] as a bone substitute for posterolateral spinal fusion also has been reported. However, the molecular and biochemical signals by which MSCs interact with their surrounding environment require further elucidation to develop improved scaffolding materials.

In the process of biological developmental, osteoblasts differentiate from mesenchymal progenitors, going through distinct developmental stages that are regulated by various developmental signals and signaling pathways. These pathways include BMP signaling [11], Hedgehog signaling [12], Notch signaling [13], Wnt signaling [14], FGF signaling [15], and leptin-leptin-R signaling [16]. Leptin is a $16-\mathrm{kDa}$ peptide hormone product of the ob (Lep) gene that is secreted by white adipose tissue [17], human osteoblasts [18], and MSCs [19]. Leptin- $\mathrm{R}$ is expressed on the surface of human bone marrow MSCs [20]. Leptin has a significant effect of promoting osteogenesis and inhibiting adipogenesis in MSCs. Runx2 messenger RNA (mRNA) expression is upregulated by leptin during osteogenesis in MSCs, while leptin reduced PPAR 2 mRNA expression during adipogenesis in MSCs $[21,22]$. Combined treatment with leptin and rhBMP-2 has a synergistic osteoinductive activity in nude mice [23]. In addition, leptin inhibits osteoclast generation [24]. Leptin increased osteoprotegerin (OPG) mRNA and protein expression in MSCs, and the inhibitory effect may be mediated by the RANKL/RANK/OPG system [24]. Leptin activity in MSCs from osteoporotic women appears hampered, suggesting that inadequate leptin activity contributes to excessive lipid accumulation in the bone marrow [21, 22].

Previous studies have employed various molecular methods to analyze gene expression in cells and tissues in contact with biomaterials and their dissolution products $[25,26]$. In the present study, we used alkaline phosphatase (ALP) activity, quantitative polymerase chain reaction (Q-PCR), and Western blotting to analyze gene and protein expression during osteogenesis of MSCs cultured in different combinations of osteoconductive materials (HA, BCP, and collagen). By comparing the expression profile of leptin, leptin-R, ALP, osteopontin (OPN), osteocalcin (OSC), and Runx2, we first proved that calcium phosphate ceramics could stimulate the differentiation of MSCs via influencing the expression of molecules in the leptin/leptin-R/ALP/OSC signaling pathway. In addition, we cultured rabbit MSCs in BCP or HA hybrid scaffolds and transplanted these mixtures into rabbits for posterior spinal fusion. We investigated the differences between $\mathrm{BCP}$ and HA hybrid scaffolds by dual-energy X-ray absorptiometry (DEXA) and computed tomography (CT) examination.

\section{Materials and methods}

The experimental protocol was approved by the human subjects Institutional Review Board and the Institutional Animal Care and Use Committee of the Chang Gung Memorial Hospital.

\section{Surgical procedures}

MSCs were harvested from patients who underwent iliac bone grafting for spine fusion. For each patient, $10 \mathrm{~mL}$ of bone marrow was aspirated and collected in a sterile heparin-rinsed syringe.

\section{Isolation and cultivation of MSCs}

Each marrow sample was washed with phosphatebuffered saline (PBS). Up to $2 \times 10^{8}$ nucleated cells in $5 \mathrm{~mL}$ of PBS were loaded onto $25 \mathrm{~mL}$ of Percoll cushion (Pharmacia Biotech). A density gradient was used as the isolation procedure to eliminate unwanted cell types. A small percentage of cells were isolated from the density interface at $1.073 \mathrm{~g} / \mathrm{mL}$. The cells were resuspended and plated in T-75 flasks at $2 \times 10^{5}$ cells per flask. The cells were maintained in Dulbecco's Modified Eagle's MediumLow Glucose (DMEM-LG; Gibco, Grand Island, NY) containing $20 \%$ fetal bovine serum (FBS) and antibiotics at $37{ }^{\circ} \mathrm{C}$ in a humidified atmosphere of $5 \% \mathrm{CO}_{2}$ and $95 \%$ air. After 7 days of primary culturing, the non-adherent cells were removed by changing the medium. The MSCs grew as symmetric colonies and were subcultured at 10 to 14 days by treatment with $0.05 \%$ trypsin (Gibco) and seeded into fresh flasks. 
Flow cytometric analysis of surface antigen expression When confluent, the MSCs were passaged 1 in 3, and a sample was analyzed by flow cytometry for MSC marker expression. The cells were washed in PBS and then removed from the flask by $0.05 \%$ trypsin (Gibco). $1 \times 10^{5}$ cells were incubated with each mouse monoclonal primary antibody at $4{ }^{\circ} \mathrm{C}$ for $30 \mathrm{~min}$. Mouse FITC-conjugated antiCD105 antibody (1:100 dilution) and mouse FITCconjugated anti-CD34 antibody (1:100 dilution) were purchased from Becton Dickinson (Oxford, UK). Mouse FITC-conjugated anti- $\alpha$ SMA antibody (1:25 dilution) was purchased from Abcam (Cambridge, UK). Mouse PEconjugated anti-STRO-1 antibody (1:50 dilution) was purchased from Santa Cruz (CA, USA). After wash, the cells were resuspended in $500 \mu \mathrm{L}$ wash buffer and analyzed on a BD flow cytometer (Oxford, UK).

\section{Construction of the PLGA-calcium phosphate-collagen carrier for MSCs}

A composite system was developed using poly(lactide-coglycolide) (PLGA) copolymer, coralline HA, BCP ceramics, and collagen as a carrier matrix for MSCs. PLGA (300 mg; Boehringer Ingelheim, Germany) and coralline HA ceramics (100 mg; Pro-Osteon 500R, Interpore International, Irvine, CA) or BCP (15 \% HA/85 \% $\beta$-TCP 100 mg; MASTERGRAFT ${ }^{\oplus}$ Granules, BioHorizons IPH Inc., USA) were loaded into molds and hot compression molded at $55{ }^{\circ} \mathrm{C}$ to form a PLGA/coralline $\mathrm{HA}$ or PLGA/BCP capsule graft. Roughly, $10^{7}$ MSCs were mixed well with $0.8 \mathrm{~mL}$ of type I collagen solution (Roche, Germany), $0.1 \mathrm{~mL}$ of $0.2 \mathrm{~mol} / \mathrm{L}$ HEPES (pH 7.3), and $0.1 \mathrm{~mL}$ of $10 \times$ concentrated complete medium (DMEM-LG) or osteogenic medium (DMEM-LG containing $100 \mathrm{mmol} / \mathrm{L}$ ascorbate-2 phosphate, $10^{-7} \mathrm{M}$ dexamethasone, $10 \mathrm{mmol} / \mathrm{L}$ $\beta$-glycerophosphate, and $20 \% \mathrm{FBS}$ ) and stored at $4{ }^{\circ} \mathrm{C}$. Approximately $250 \mu \mathrm{L}$ of the MSC-collagen mixture was loaded into the cores of a PLGA/coralline HA or PLGA/ $\mathrm{BCP}$ capsule and incubated at $37^{\circ} \mathrm{C}$ for $8 \mathrm{~h}$ to enable collagen gel formation. The composite systems were cultured in six-well plates containing $2.5 \mathrm{~mL}$ of complete medium or osteogenic medium and incubated at $37^{\circ} \mathrm{C}$. The medium was changed per $72 \mathrm{~h}$.

\section{Quantitative measurement of ALP activity}

After culturing for 14 days, the culture medium was withdrawn and the MSC carrier was washed twice with $5 \mathrm{~mL}$ of Tyrode's balanced salt solution. A $5-\mathrm{mL}$ aliquot of ALP substrate buffer $(50 \mathrm{mmol} / \mathrm{L}$ glycine, $1 \mathrm{mmol} / \mathrm{L}$ $\mathrm{MgCl}_{2}, \mathrm{pH}$ 10.5), containing the soluble chromogenic ALP substrate $(2.5 \mathrm{mmol} / \mathrm{L} p$-nitrophenyl phosphate), was added at room temperature. During incubation, ALP converts $p$-nitrophenyl phosphate into $p$-nitrophenol, which is yellow in color. Twenty minutes after substrate addition, $1 \mathrm{~mL}$ of the buffer was withdrawn and mixed with $1 \mathrm{~mL}$ of $1 \mathrm{~N} \mathrm{NaOH}$ to stop the reaction. The absorbance of the mixture was read in triplicate at $405 \mathrm{~nm}$ on an ELISA MRX plate reader (Dynatech Labs, Chantilly, VA). Enzyme activity was expressed as nanomoles $p$-nitrophenol per minute per dish.

\section{RNA preparation and Q-PCR analysis}

After culturing for 7 or 14 days, total RNA was extracted using a Qiagen RT kit (Qiagen, USA) according to the manufacturer's instructions. The RNA concentration was evaluated by A260/A280 measurement. To detect leptin-R, OPN, OSC, Runx2, and glyceraldehyde 3-phosphate dehydrogenase (GAPDH) RNA transcripts, cDNA was analyzed on an ABI PRISM 7900 sequence detection system using TaqMan PCR Master Mix (Applied Biosystems, Foster City, CA). The cycle threshold $(\mathrm{Ct})$ values were obtained, and the data were normalized to GAPDH expression by using the $\Delta \Delta \mathrm{Ct}$ method to calculate the relative mRNA level of each gene.

\section{Western blot analysis}

After culturing for 7 or 14 days, the cells were washed with PBS and extracted using M-PER protein extraction reagent (Thermo, USA). The protein content was quantitated using a protein assay kit (Pierce Biotechnology, IL), separated by $7.5 \%$ SDS-PAGE, and transferred onto membranes using a transfer unit (Bio-Rad, USA). After blocking, the membranes were incubated with 1000-fold diluted rabbit antibodies against leptin (R\&D, MN, USA), leptin-R (R\&D), Runx2 (Millipore, Temecula, CA), and $\beta$-actin (Millipore). After washing, the membranes were further incubated for $2 \mathrm{~h}$ with 10,000-fold goat antimouse IgG (Calbiochem, USA) or goat anti-rabbit IgG (Millipore) conjugated to horseradish peroxidase. The membranes were then washed and rinsed with ECL detection reagents (Millipore). The bands were photographed using ECL Hyperfilm (Amersham Pharmacia Biotech, UK), and their intensity was quantified using an image analysis system (Image-Pro plus 5.0).

\section{Microarray analysis}

After culturing, cells were collected for studying the differential gene expression pattern using Affymetrix Human Genome U133 plus microarrays (Affymetrix, Santa Clara, CA) according to the manufacturer's protocol. RNA preparation was performed using Qiagen's RNeasy Mini Kit, and the integrity and purity were analyzed using Agilent Bioanalyzer and NanoDrop spectrophotometer. Raw gene expression data were normalized and analyzed with GeneChip Operating Software 1.4 (GCOS, Affymetrix). Genes that are $>1.5$-fold up- or downregulated between two groups were selected. 


\section{Animal experiments and operative procedures}

Six New Zealand white rabbits, each about 8 months old and weighing 3.3 to $3.8 \mathrm{~kg}$, were used. Under anesthesia, a 6-cm dorsal midline incision was made to expose the bilateral paramedian fascia. The intermuscular plane between the multifidus and longissimus muscles was dissected to expose the intertransverse space between L4 and L5. The soft tissues between the L4 and L5 transverse processes were dissected, and decortication of the transverse processes was performed by using an electric burr. An MSC/PLGA/BCP/collagen graft was placed on the left side and a MSC/PLGA/coralline HA/collagen graft was placed on the right side between the transverse processes in the paraspinal bed. The fascia and skin incisions were sutured. The rabbits were killed at 10 weeks after grafting and underwent radiographic, $\mathrm{CT}$, and bone mineral content (BMC) examinations.

\section{Computed tomography examination}

At 10 weeks after grafting, three rabbits underwent high-resolution helical CT scanning at a $2.0-\mathrm{mm}$ slice resolution in $1.0-\mathrm{mm}$ increments. CT images were transferred to a workstation and reconstructed threedimensionally. Multiple slices obtained in three planes were reviewed for each fusion mass.

\section{Dual-energy X-ray absorptiometry examination}

At 10 weeks after grafting, three rabbits underwent BMC measurements by using a Hologic QDR 2000 dualenergy X-ray absorptiometry system (Hologic, Inc., Boston, MA). A self-contained X-ray source was mounted beneath an animal to provide alternating pulses at 70 and $140 \mathrm{kVp}$. An X-ray detector was then mounted above the animal, and both source and detector were moved across the animal under computer control in a serpentine pattern. The two radiation energies were generated as collimated beams and their absorption patterns allowed for the measurement of $\mathrm{BMC}$ in grams.

\section{Results}

In vitro studies

\section{Flow cytometry analysis}

Primary adherent human MSCs from three donors were cultured in a control medium, and the cells were analyzed for expression of MSC markers using flow cytometry at passage 1 . The percentage of cells expressing the MSC markers CD105, Stro-1, and $\alpha$-SMA and the hematopoietic stem cell (HSC) marker CD34 are shown in Fig. 1. The mean percentages of $\mathrm{CD} 105^{+}$, Stro- $1^{+}$, $\alpha-\mathrm{SMA}^{+}$, and $\mathrm{CD}^{+} 4^{+}$cells in the cell preparations from three patients were calculated to be $85.7 \pm 5.7$, $32.7 \pm 4.9,53.6 \pm 1.8$, and $0.21 \pm 0.07 \%$, respectively.

\section{Gene expression profiling}

MSCs cultured in different scaffolds modulate the expression of several genes, leading to higher expression of 80 genes (BCP hybrid scaffold/coralline HA hybrid scaffold ratio $>1.5$-fold) and lower expression of 101 genes (BCP hybrid scaffold/coralline HA hybrid scaffold ratio <0.67-fold) in BCP hybrid scaffolds in 22,215 human genes. The top 12 higher and lower gene expressions of MSCs cultured in BCP hybrid scaffolds are shown in Table 1.

\section{mRNA expression of osteogenic genes and leptin- $R$}

Figure 2 presents the mRNA expression of osteogenic genes and leptin- $\mathrm{R}$ in the MSCs. After 7 days of culture, the mRNA levels of leptin-R $(1.44 \pm 0.23$-fold, * $p<0.05, n=3)$, osteopontin (1.65 \pm 0.38 -fold, * $p<0.05, n=3$ ), and Runx2 (1.43 \pm 0.2 -fold, ${ }^{*} p<0.05, n=3$ ) were significantly higher in the PLGA/BCP-group MSCs than in the PLGA/ coralline-HA-group MSCs. After 14 days of culture, the mRNA levels of leptin-R $\left(2.27 \pm 0.36\right.$-fold, ${ }^{* *} p<0.01$, $n=3)$, osteopontin (3.18 \pm 0.55 -fold, $\left.{ }^{* * *} p<0.01, n=3\right)$, osteocalcin (1.83 \pm 0.34 -fold, $* p<0.05, n=3$ ), and Runx2 $\left(2.28 \pm 0.29\right.$-fold, $\left.{ }^{* * *} p<0.01, n=3\right)$ were significantly higher in the PLGA/BCP-group MSCs than in the PLGA/ coralline-HA-group MSCs.

\section{Osteogenic protein expression}

Figure 3 presents the Western blotting results for the expression of osteogenic proteins in the MSCs. Compared to a control group, the expression of the detected osteogenic protein in the coralline-HA-group MSCs and in the BCP-group MSCs was considerably enhanced. After 14 days of culture, the protein levels of leptin-R (BC14/ BO14 $0.12 \pm 0.03$-fold, $* p<0.01, n=3$ ), leptin (BC14/ BO14 $0.70 \pm 0.06$-fold, $* p<0.05, n=3$ ), and Runx2 (BC14/BO14 $0.65 \pm 0.07$-fold, ${ }^{*} p<0.05, n=3$ ) were significantly higher in the $\mathrm{BCP} /$ osteogenic-group MSCs than in the $\mathrm{BCP} /$ control-group MSCs. Additionally, the protein levels of leptin-R (HO14/BO14 0.24 \pm 0.04-fold, ** $p<0.01, n=3$ ) and Runx2 (HO14/BO14 $0.61 \pm 0.04-$ fold, $* p<0.01, n=3$ ) were significantly higher in the $\mathrm{BCP} /$ osteogenic-group MSCs than in the coralline-HA/ osteogenic-group MSCs.

\section{Quantitative measurement of ALP}

Figure 4 presents the results of ALP activity measurements. MSCs cultured in a PLGA/coralline HA hybrid scaffold or PLGA/BCP hybrid scaffold had significantly lower ALP activity levels in the control (complete) medium than in the osteogenic medium throughout the 14-day culture period. In addition, ALP activity levels in the PLGA/BCP-group MSCs cultured in osteogenic medium were significantly higher than those in the PLGA/coralline-HA-group MSCs throughout the 14-day 

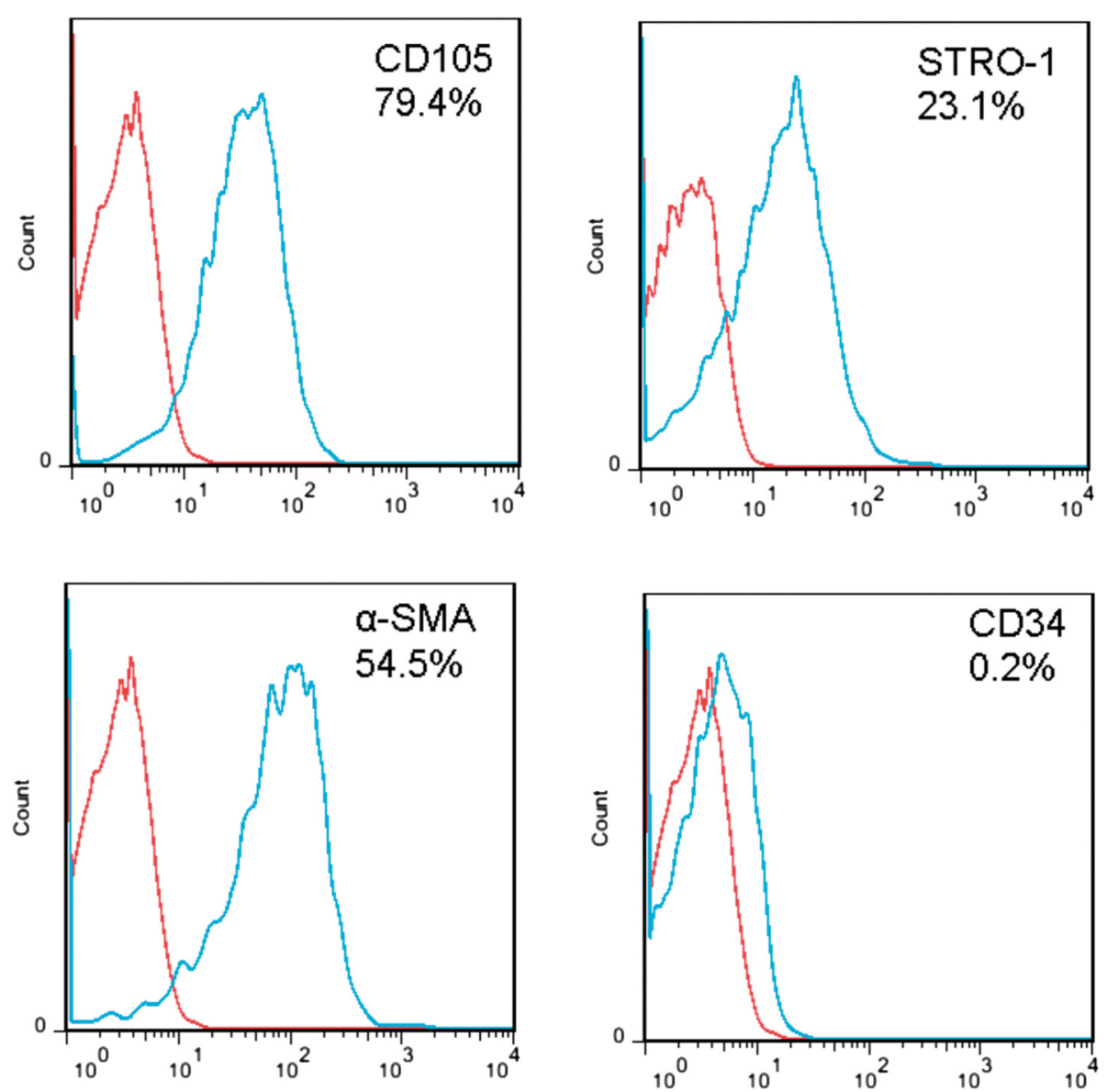

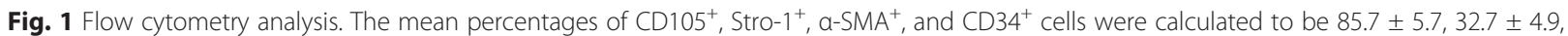
$53.6 \pm 1.8$, and $0.21 \pm 0.07 \%$, respectively

culture period (PLGA/coralline HA vs. PLGA/BCP $1.57 \pm 0.21$ vs. $2.53 \pm 0.4, p<0.05, n=3)$.

\section{In vivo studies}

\section{Computed tomography findings}

3D CT reconstructions revealed the presence of continuous bone bridges and satisfactory fusion mass incorporated with the transverse processes (Fig. 5a, b).

\section{Dual-energy X-ray absorptiometry examination}

Radiographs of the specimens retrieved from the BCP graft and coralline HA graft showed continuous trabecular bone patterns in the area between the transverse processes (Fig. 6a). The BMC values were higher in the BCP graft than in the coralline HA graft (Fig. 6b; BCP vs. coralline HA $0.36 \pm 0.05$ vs. $0.26 \pm 0.03 \mathrm{~g},{ }^{*} p<0.05, n=3$ ).

\section{Discussion}

A recent study proposed the theory that certain materials could trigger the secretion of factors leading to bone formation rather than simply causing the accumulation of such factors [27]. Other studies have focused on the direct effects of different materials on various types of multipotential cells that can eventually differentiate into osteocytes, such as adipose-derived stem cells [28], C2C12 cells [29], and bone marrow MSCs [30]. The MSCs used here were characterized by the presence of a consistent set of marker proteins on their surface. The percentages of cells expressing the human MSC markers CD105, Stro-1, and $\alpha$-SMA are shown in Fig. 1.

Up to now, a series of biomaterials have been reported to be osteoinductive, including demineralized bone matrix [16], porous HA ceramics [31], and TCP ceramics [3]. Although these findings expanded the understanding of osteoinductive biomaterials, the osteoinductive mechanism of those biomaterials behind the phenomenon of ectopic formation is still not fully understood. Tan et al. observed that $\mathrm{HA}$ and $\mathrm{BCP}$ induced the expression of osteogenic markers in $\mathrm{C} 2 \mathrm{C} 12$ cells, but they did not quantify the differences between the two ceramics [29]. Barradas et al. showed that MSCs cultured in $\beta$-TCP express more OPN, OC, collagen type I ( $\mathrm{Col} \mathrm{I})$, and bone sialoprotein (BSP) than MSCs cultured in HA after 7 days [32]. In the present study, coralline HA hybrid scaffold 
Table 1 Microarray analyses of the top 12 higher and lower gene expressions of MSCs cultured in BCP hybrid scaffolds

\begin{tabular}{|c|c|c|c|}
\hline Gene symbol & Entrez Gene & $\begin{array}{l}\text { RefSeq transcript } \\
\text { ID }\end{array}$ & $\begin{array}{l}\text { BCP hybrid scaffold/ } \\
\text { HA hybrid scaffold } \\
\text { fold changes }\end{array}$ \\
\hline \multicolumn{4}{|l|}{ (a) Higher } \\
\hline LEPR & 3953 & $\begin{array}{l}\text { NM_001003679 /// } \\
\text { NM_001003680 /// } \\
\text { NM_002303 }\end{array}$ & 4.25 \\
\hline MAFB & 9935 & NM_005461 & 2.99 \\
\hline IFI16 & 3428 & NM_005531 & 2.81 \\
\hline FLJ10159 & 55084 & NM_018013 & 2.35 \\
\hline MMP13 & 4322 & NM_002427 & 2.27 \\
\hline $\mathrm{COL} 21 \mathrm{~A} 1$ & 81578 & NM_030820 & 2.09 \\
\hline EIF4A1 & 1973 & NM_001416 & 2.02 \\
\hline SEPP1 & 6414 & NM_005410 & 1.95 \\
\hline APOE & 348 & NM_000041 & 1.94 \\
\hline RUNX2 & 860 & $\begin{array}{l}\text { NM_001015051 /// } \\
\text { NM_001024630 /// } \\
\text { NM_004348 }\end{array}$ & 1.94 \\
\hline PTGFR & 5737 & NM_000959 & 1.93 \\
\hline OLFML2B & 25903 & NM_015441 & 1.89 \\
\hline \multicolumn{4}{|l|}{ (b) Lower } \\
\hline INHBA & 3624 & NM_002192 & 0.50 \\
\hline WSB1 & 26118 & $\begin{array}{l}\text { NM_015626 /// } \\
\text { NM_134264 /// } \\
\text { NM_134265 }\end{array}$ & 0.49 \\
\hline GOLGA8A & 23015 & NM_015003 & 0.48 \\
\hline STMN2 & 11075 & NM_007029 & 0.48 \\
\hline RIS1 & 25907 & NM_015444 & 0.48 \\
\hline PPAP2B & 8613 & $\begin{array}{l}\text { NM_003713 /// } \\
\text { NM_177414 }\end{array}$ & 0.47 \\
\hline PWP1 & 11137 & NM_007062 & 0.46 \\
\hline IQGAP1 & 8826 & NM_003870 & 0.43 \\
\hline COMP & 1311 & NM_000095 & 0.41 \\
\hline RGC32 & 28984 & NM_014059 & 0.38 \\
\hline STC1 & 6781 & NM_003155 & 0.36 \\
\hline PGK1 & 5230 & NM_000291 & 0.33 \\
\hline
\end{tabular}

and $\mathrm{BCP}$ hybrid scaffold were also found to upregulate the protein expression of osteogenic markers of MSCs in the osteogenic medium (Fig. 3). In addition, we found higher mRNA expression of OPN, Runx2, and OSC (Fig. 2); higher protein levels of leptin, leptin-R, and Runx2 (Fig. 3); and higher ALP activity (Fig. 4) in MSCs grown in the PLGA/BCP hybrid scaffold which were cultured in the osteogenic medium. All of the above findings make it more reasonable to conclude that the osteoinductive feature of these materials might rely on their ability to directly induce the differentiation of MSCs along an osteoblastic lineage.

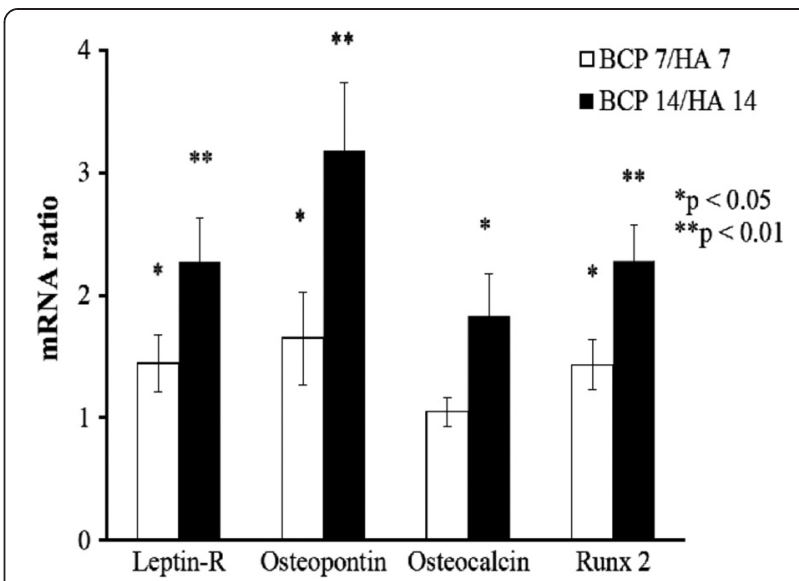

Fig. 2 mRNA expression of osteogenic genes and leptin-R. After 7 days of culture, the mRNA levels of leptin- $R\left({ }^{*} p<0.05, n=3\right)$, osteopontin $\left({ }^{*} p<0.05, n=3\right)$, and Runx2 $\left({ }^{*} p<0.05, n=3\right)$ were significantly higher in the PLGA/BCP-group MSCs than in the PLGA/ coralline-derived-HA-group MSCs. After 14 days of culture, the mRNA levels of leptin-R $\left({ }^{* *} p<0.01, n=3\right)$, osteopontin $\left({ }^{* *} p<0.01, n=3\right)$, osteocalcin $\left({ }^{*} p<0.05, n=3\right)$, and Runx2 $\left({ }^{* *} p<0.01, n=3\right.$ ) were significantly higher in the PLGA/BCP-group MSCs than in the PLGA/coralline-derived-HA-group MSCs

Several signaling pathways and molecules have been shown to be critical for MSC differentiation along an osteogenic lineage [33, 34]. To explore the critical molecules initiating the osteoblastic differentiation of cells stimulated by calcium phosphate materials, Tang et al.

\section{B014 BC14 HO14 HC14}

\section{Leptin-R}

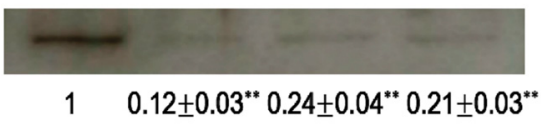

Leptin

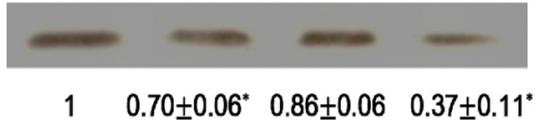

Runx 2

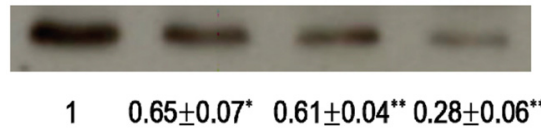

\section{$\beta$-actin}

Fig. 3 Osteogenic protein expression. Compared to a control group, the expression of the detected osteogenic proteins in the coralline-derived-HA-group MSCs and in the BCP-group MSCs was considerably enhanced. After 14 days of culture, the protein levels of leptin-R (** $p<0.01, n=3$ ), leptin (0.70 \pm 0.06 -fold, ${ }^{*} p<0.05$, $n=3)$, and Runx2 ( ${ }^{*} p<0.05, n=3$ ) were significantly higher in the $\mathrm{BCP} /$ osteogenic-group MSCs than in the BCP/control-group MSCs. Additionally, the protein levels of leptin- $R\left({ }^{* *} p<0.01, n=3\right)$ and Runx2 $\left({ }^{* *} p<0.01, n=3\right)$ were significantly higher in the BCP/osteogenic-group MSCs than in the coralline-derived-HA/osteogenic-group MSCs 


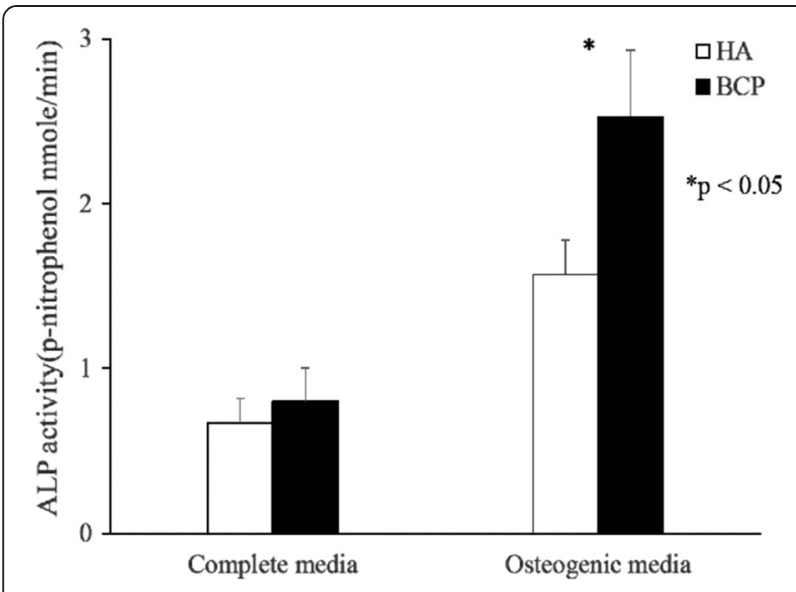

Fig. 4 Quantitative measurement of ALP. MSCs cultured in PLGA/ coralline-derived HA hybrid scaffolds or PLGA/BCP hybrid scaffolds had significantly lower ALP activity levels in the control (complete) medium than in the osteogenic medium throughout the 14-day culture period. In addition, ALP activity levels in the PLGA/BCP-group MSCs cultured in the osteogenic medium were significantly higher than those in the PLGA/coralline-derived-HA-group MSCs throughout the 14 -day culture period $\left({ }^{*} p<0.05, n=3\right)$

proved that the osteoinductive calcium phosphates, both $\mathrm{HA}$ and $\mathrm{BCP}$, could upregulate the gene expression of BMPs, in addition to even upregulating the expression of intracellular signal transduction molecules in the Smad pathway during osteogenesis in MSCs [35]. Leptin exerts its effect by binding to leptin- $\mathrm{R}$ and activating the JAK/STAT pathway, which is highly expressed in human osteoblasts $[36,37]$. In the present study, the BCP hybrid scaffold culture resulted in higher upregulation of mRNA (Fig. 2) and protein (Fig. 3) expression of leptin$\mathrm{R}$ in MSCs than did the coralline HA hybrid scaffold. Leptin [19] and leptin-R [20] are expressed in MSCs and might act as a local (autocrine) factor in the osteogenesis of MSCs and bone remodeling. Enhancement of the expression of transforming growth factor (TGF)- $\beta$ [38] and ALP [39] by leptin has previously been reported, and the stimulatory effect of leptin on the osteogenesis of MSCs was also confirmed by the ALP activity measured in this study (Fig. 4).

Resorbable devices have been used in applications such as fracture fixation, bone grafting, spinal fusion, and deformity correction. A previous study showed the HA-grafted implants were poorly anchored compared with allografted implants, suggesting that coralline HA granules should be considered a bone graft extender rather than a bone graft substitute [40]. Coralline-derived HA typically performs in an inferior manner to other HA materials in implant fixation. However, corallinederived HA representing as a promising bone graft extender in spinal fusion has also been reported [41]. In addition, a system review showed the overall fusion rate for all ceramic products (including $\beta$-TCP, BCP, calcium sulfate, coralline hydroxyapatite, synthetic hydroxyapatite, silicated hydroxyapatite) as a bone graft extender in the lumbar spine was $86.4 \%$ in 30 studies with 1332 patients. The data for coralline hydroxyapatite (Pro-Osteon 200, Pro-Osteon 500) was 86.9 \% in 7 studies with 168 patients. The coralline-derived HA typically performs in a similar manner to other HA materials in the lumbar spinal fusion [42]. In our previous study, we showed that the MSC/coralline-derived HA/type I collagen hybrid graft could be effectively used to achieve posterolateral spinal fusion [9]. In this study, we further investigated the gene, mRNA, and protein expression of MSCs cultured on BCP hybrid scaffolds and corallinederived HA hybrid scaffolds (Figs. 2, 3, and 4). In addition, we put these MSC-BCP hybrid scaffolds and MSC-coralline-derived HA hybrid scaffolds into rabbits for spinal fusion test (Figs. 5 and 6).

Previous studies demonstrated that HA/TCP stimulated the osteogenic differentiation of MSCs [43, 44]. In this study, BCP hybrid scaffolds were performed using MASTERGRAFT, a commercial composite consisting of $15 \%$ HA and $85 \% \beta$-TCP. HA is a material with biomechanical properties similar to natural bone. Briefly, when compared to $\beta$-TCP, HA is a more stable phase under physiological conditions, as it has a lower solubility and, thus, slower resorption kinetics $[45,46]$. Therefore, the $\mathrm{BCP}$ concept is determined by the optimum balance of a more stable phase of HA and a more soluble TCP. Due to a higher biodegradability of the $\beta$-TCP component, the reactivity of $\mathrm{BCP}$ increases with the $\mathrm{TCP} / \mathrm{HA}$ ratio increasing.

Differences in the porosity and surface area of particles within the material can influence its performance characteristics. Porosity is defined as a percentage of voids in solids and this morphological property is independent of the material. The surface area of porous bodies is much higher, which guarantees a good mechanical fixation in addition to providing sites on the surface that allow chemical bonding between the bioceramics and bones [47]. In addition, pore dimensions are also important. The dimensions of open pores are directly related to bone formation, since such pores grant both the surface and space for cell adhesion and bone ingrowth [48]. In this study, coralline-derived HA hybrid scaffolds were performed using the commercial product Pro-Osteon 500 . Pro-Osteon 500 is prepared by the hydrothermal conversion $\left(260{ }^{\circ} \mathrm{C}, 15,000 \mathrm{psi}\right)$ of coral (consisting of mostly $\mathrm{CaCO}_{3}$, calcite form) in the presence of ammonium phosphate to hydroxyapatite [49]. Pro-Osteon 500 has a porosity rate of $60-70 \%$ and a mean pore size of $500 \mu \mathrm{m}$. BCP hybrid scaffolds were performed using MASTERGRAFT. $\mathrm{BCP}$ of varying HA to $\beta$-TCP weight ratios is obtained by sintering precipitated calcium-deficient 


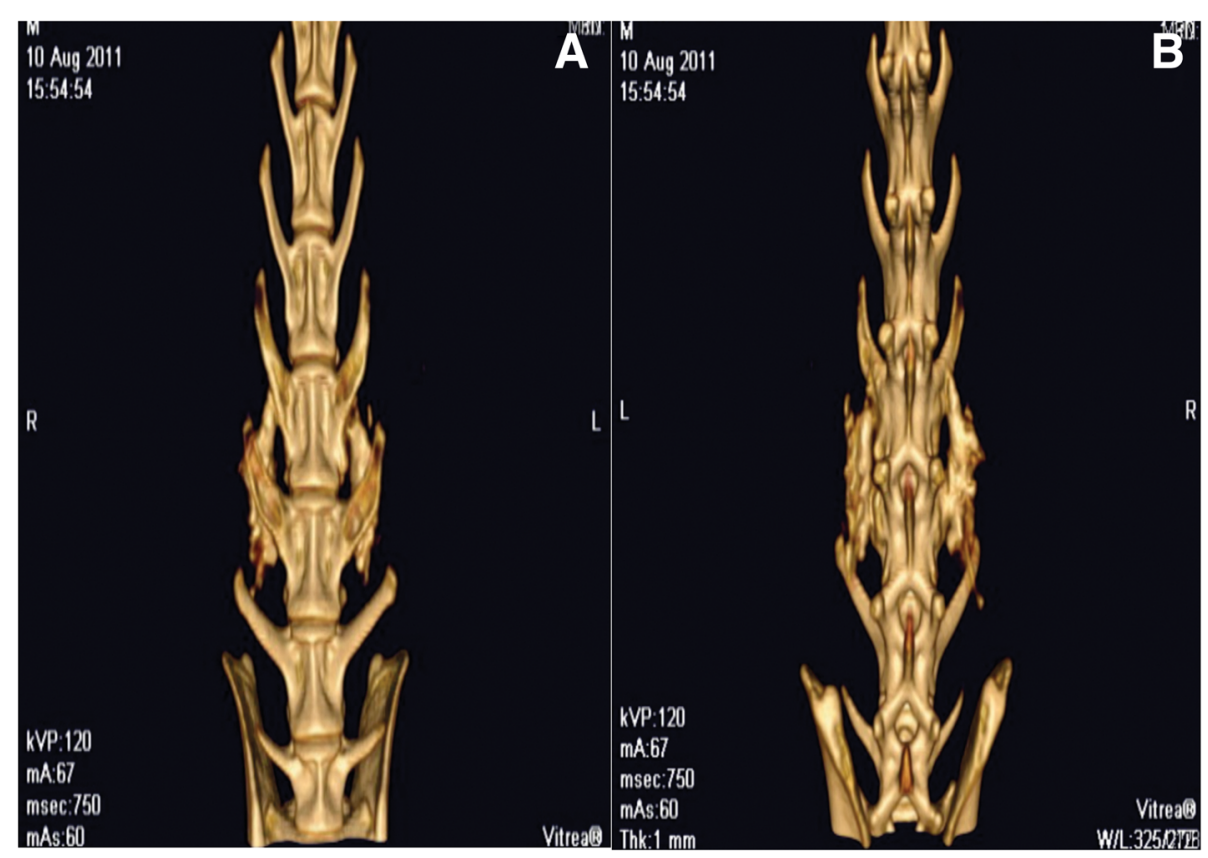

Fig. 5 Computed tomography findings. Three-dimensional $C T$ reconstructions revealed the presence of continuous bone bridges and satisfactory fusion mass incorporated with the transverse processes $(\mathbf{a}, \mathbf{b})$

apatite $(\mathrm{Ca} / \mathrm{P}$ molar ratio $<1.67)$. MASTERGRAFT has a porosity rate of $80 \%$ and a mean pore size of $500 \mu \mathrm{m}$. The mean pore size between the two calcium phosphate materials is equal, but the porosity rate is higher in MASTERGRAFT than in Pro-Osteon 500. This may be one of the reasons why the BMC values were higher in BCP hybrid scaffolds than in coralline-HA hybrid scaffolds (Fig. 6).
To analyze the osteoinductive potential of ceramics in vivo, a previous study conducted ectopic implantation in the muscle tissue and femoral cortical bones of dogs [50]. In muscle, histomorphometric analysis showed earlier and more bone formed in BCP than in HA. In femoral cortical bone defects, significantly more bone was formed in BCP than in HA. Similarly, a comparison between $\mathrm{HA}$ and BCP sintered at the same temperature

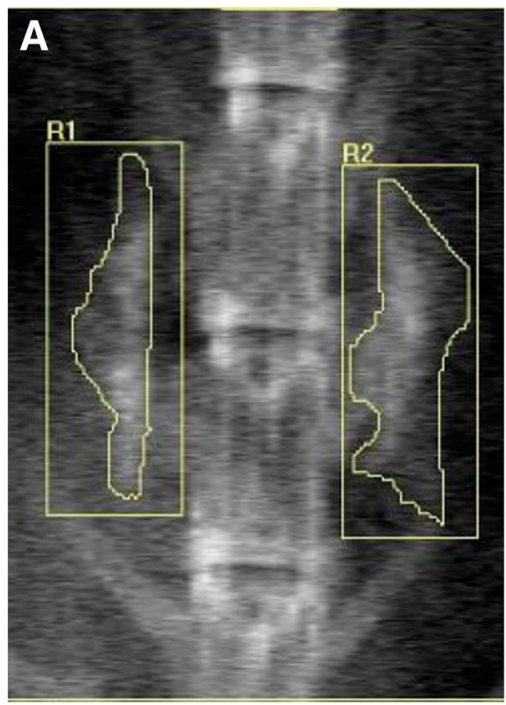

B

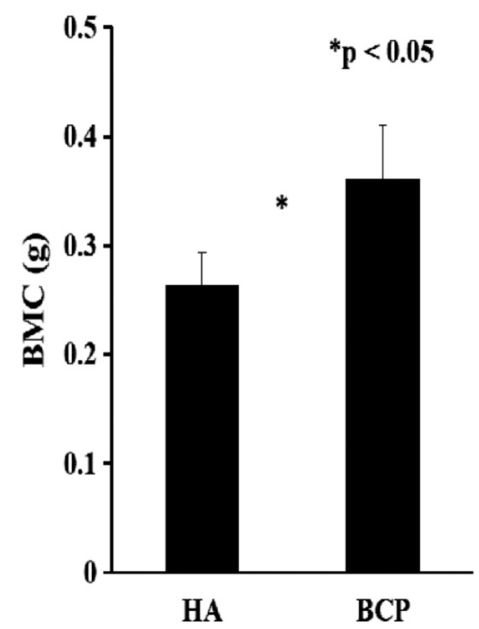

Fig. 6 Dual-energy X-ray absorptiometry examination. Radiographs of the specimens retrieved from the BCP graft and HA graft showed continuous trabecular bone patterns in the area between the transverse processes (a). The BMC values were higher in the BCP graft than in the coralline-derived HA graft $\left(\mathbf{b} ;{ }^{*} p<0.05, n=3\right)$ 
(thus with similar macro- and microstructural features but different chemistry) showed a more pronounced bone formation in BCP [51]. Our previous study showed that an MSC/coralline HA/type I collagen hybrid graft could be effectively used to achieve posterolateral spinal fusion in a rabbit model [9]. In the present study, we used the clinically relevant posterolateral spinal fusion model in which two materials can be compared in a paired manner. In our results, 3D reconstructed CT images showed continuous bone bridges and fusion mass incorporated with the transverse processes (Fig. 5). BMC values were higher in $\mathrm{BCP}$ hybrid scaffolds than in coralline HA hybrid scaffolds (Fig. 6). These results indicated that the osteogenic capacities of osteoinductive materials in osseous fusion sites are correlated: the higher the osteoinductive potential of the material, the faster the formation of the bone bridges.

To provide more information, we show the microarray analysis of MSCs cultured on coralline-derived HA hybrid scaffolds and BCP hybrid scaffolds in Table 1. Higher levels of osteogenic marker expression in BCP hybrid scaffolds were shown (Runx2 and leptin-R). In addition, higher levels of MAFB and IQGAP1 and lower levels of COMP expression in BCP hybrid scaffolds were shown. MAFB (v-maf musculoaponeurotic fibrosarcoma oncogene homolog B) is a biomarker for rheumatoid arthritis [52]. IQGAP1 (IQ motif containing GTPase activating protein 1 ) modulates many different signaling pathways and cellular functions, including mitogen-activated protein kinase (MAPK) signaling, $\mathrm{Ca}^{2+} /$ calmodulin signaling, cell-cell adhesion, $\beta$-catenin-mediated transcription, and microbial invasion [53]. COMP (cartilage oligomeric matrix protein) is a biomarker for cartilage degradation [54]. However, the role of MAFB, IQGAP1, and COMP in osteogenesis of MSCs needs to be further investigated.

In conclusion, the results of the present study indicate that a BCP culture is more effective than an HA culture at inducing the osteogenesis of MSCs due to the greater capacity of the $\mathrm{BCP}$ culture to upregulate the expression of leptin receptors and even the intracellular signal transduction molecules of osteogenesis of MSCs. This is correlated to in vivo data, where $\mathrm{BCP}$ induced more bone formation in a rabbit spinal fusion model.

\footnotetext{
Abbreviations

MSCs: Mesenchymal stem cells; HA: Hydroxyapatite; BCP: Biphasic calcium phosphate; PLGA: Poly(lactide-co-glycolide); ALP: Alkaline phosphatase; Q-PCR: Quantitative polymerase chain reaction; CT: Computed tomography; DEXA: Dual-energy X-ray absorptiometry; Leptin-R: Leptin receptor; BMC: Bone mineral content; HSC: Hematopoietic stem cell; BSP: Bone sialoprotein; MAFB: V-maf musculoaponeurotic fibrosarcoma oncogene homolog B; IQGAP1: IQ motif containing GTPase activating protein 1; COMP: Cartilage oligomeric matrix protein, biomarker for cartilage degradation.
}

\section{Competing interests}

The authors declare that they have no competing interests.

\section{Authors' contributions}

WJC, SSL, CCN, and SJL designed the study. LJY, CYY, CJW, and HYC analyzed the data and interpreted the results. CCN, WJC, and LHC provided the study materials or patients. SSL and PHC drafted the manuscript. All authors approved the final version of the manuscript.

\section{Acknowledgements}

This research was supported in part by grants from the National Science Council and Chang Gung Memorial Hospital (CMRPG3C0081 2), Taiwan, Republic of China.

\section{Author details}

${ }^{1}$ Department of Orthopaedics, Chang Gung Memorial Hospital, No. 5, Fu-Hsing Street 333, Kweishan, Taoyuan, Taiwan. ${ }^{2}$ College of Medicine, Chang Gung University, Taoyuan, Taiwan. ${ }^{3}$ Department of Mechanical Engineering, Chang Gung University, Taoyuan, Taiwan. ${ }^{4}$ Department of Radiology, Chang Gung Memorial Hospital, Taoyuan, Taiwan.

Received: 17 March 2015 Accepted: 12 June 2015

Published online: 16 July 2015

\section{References}

1. Fernyhough JC, Schimandle JH, Weigel MC, Edwards CC, Levine AM. Chronic donor site pain complicating bone graft harvesting from the posterior iliac crest for spinal fusion. Spine. 1992;17:1474-80.

2. Artzi Z, Weinreb M, Carmeli G, Lev-Dor R, Dard M, Nemcovsky CE. Histomorphometric assessment of bone formation in sinus augmentation utilizing a combination of autogenous and hydroxyapatite/biphasic tricalcium phosphate graft materials: at 6 and 9 months in humans. Clin Oral Implants Res. 2008;19:686-92.

3. Yuan H, Fernandes H, Habibovic P, de Boer J, Barradas AMC, de Ruiter A, et al. Osteoinductive ceramics as a synthetic alternative to autologous bone grafting. PNAS. 2010;107:13614-9.

4. Yang C, Unursaikhan O, Lee JS, Jung UW, Kim CS, Choi SH.

Osteoconductivity and biodegradation of synthetic bone substitutes with different tricalcium phosphate contents in rabbits. J Biomed Mater Res Part B. 2014;102B:80-8.

5. Lin SS, Ueng SWN, Niu CC, Yuan LJ, Yang CY, Chen WJ, et al. Hyperbaric oxygen promotes osteogenic differentiation of bone marrow stromal cells by regulating $W n+3 a / \beta$-catenin signaling - an in vitro and in vivo study. Stem Cell Res. 2014;12:260-74.

6. Pittenger MF, Mackay AM, Beck SC, Jaiswal RK, Douglas R, Mosca JD, et al. Multilineage potential of adult human mesenchymal stem cells. Science. 1999;284:143-7.

7. Yoshikawa T, Ohgishi H, Tamai S. Immediate bone forming capability of prefabricated osteogenic hydroxyapatite. J Biomed Mater Res. 1996:32:481-92.

8. Livingston Arinzeh T, Peter S, Archambault M, Van Den Bos C, Gordon S, et al. Allogeneic mesenchymal stem cells regenerate bone in a critical-sized canine segmental defect. J Bone Joint Surg Am. 2003;85A:1927-35.

9. Huang JW, Lin SSMS, Chen LH, Liu SJ, Niu CC, Yuan LJ, et al. The use of fluorescence-labeled mesenchymal stem cells in poly(lactide-co-glycolide)/ hydroxyapatite/collagen hybrid graft as a bone substitute for posterolateral spinal fusion. J Trauma. 2011;70:1495-502.

10. Kai T, Guo SQ, Dang GT. In vivo evaluation of bone marrow stromal-derived osteoblasts-porous calcium phosphate ceramic composites as bone graft substitute for lumbar intervertebral spinal fusion. Spine. 2003;28:1653-8.

11. Chen G, Deng C, Li YP. TGF-beta and BMP signaling in osteoblast differentiation and bone formation. Int J Biol Sci. 2012;8:272-88.

12. St-Jacques $B$, Hammerschmidt M, McMahon AP. Indian hedgehog signaling regulates proliferation and differentiation of chondrocytes and is essential for bone formation. Genes Dev. 1999;13:2072-86.

13. Hilton MJ, Tu X, Wu X, Bai S, Zhao H, Kobayashi T, et al. Notch signaling maintains bone marrow mesenchymal progenitors by suppressing osteoblast differentiation. Nat Med. 2008;14:306-14.

14. Lin GL, Hankenson KD. Integration of BMP, Wnt, and notch signaling pathways in osteoblast differentiation. J Cell Biochem. 2011;112:3491-501.

15. Ornitz DM, Marie PJ. FGF signaling pathways in endochondral and intramembranous bone development and human genetic disease. Genes Dev. 2002;16:1446-65. 
16. Takikawa S, Bauer TW, Kambic H, Togawa D. Comparative evaluation of the osteoinductivity of two formulations of human demineralized bone matrix. J Biomed Mater Res A. 2003;65:37-42.

17. Zhang Y, Proenca R, Maffei M, Barone M, Leopold L, Friedman JM. Positional cloning of the mouse obese gene and its human homologue. Nature. 1994;372:425-32.

18. Reseland JE, Syversen U, Bakke I, Qvigstad G, Eide LG, Hjertner O, et al. Leptin is expressed in and secreted from primary cultures of human osteoblasts and promotes bone mineralization. J Bone Miner Res. 2001;16:1426-33.

19. Noh M. Interleukin-17A increases leptin production in human bone marrow mesenchymal stem cells. Biochem Pharmacol. 2012:83:661-70.

20. Hess R, Pino AM, Rios S, et al. High affinity leptin receptors are present in human mesenchymal stem cells (MSCs) derived from control and osteoporotic donors. J Cell Biochem. 2005;94:50-7.

21. Astudillo P, Rios S, Pastenes L, Pino AM, Rodriguez JP. Increased adipogenesis of osteoporotic human-mesenchymal stem cells (MSCs) characterizes by impaired leptin action. J Cell Biochem. 2008;103:1054-65.

22. Rodriguez JP, Astudillo P, Rios S, et al. Involvement of adipogenic potentia of human bone marrow mesenchymal stem cells (MSCs) in osteoporosis. Curr Stem Cell Res Ther. 2008:3:208-18.

23. Xu JC, Wu GL, Liu HL, et al. The effect of leptin on the osteoinductive activity of recombinant human bone morphogenetic protein-2 in nude mice. Saudi Med J. 2010;31:615-21.

24. Holloway WR, Coller FM, Aitken CJ, et al. Leptin inhibits osteoclast generation. J Bone Miner Res. 2002;17:200-9.

25. Xynos ID, Edgar AJ, Buttery LDK, Hench LL, Polak JM. Gene-expression profiling of human osteoblasts following treatment with the ionic products of Bioglass 45S5 dissolution. J Biomed Mater Res. 2001:55:151-7.

26. Granjeaud S, Bertucci F, Jordan BR. Expression profiling: DNA arrays in many guises. BioEssays. 1999;21:781-90

27. Barradas A, Yuan H, Blitterswijk CA, Habibovic P. Osteoinductive biomaterials: current knowledge of properties, experimental models and biological mechanisms. Eur Cells Mater. 2011;21:407-29.

28. Barbieri D, Yuan H, de Groot F, Walsh WR, de Bruijn JD. Influence of different polymeric gels on the ectopic bone forming ability of an osteoinductive biphasic calcium phosphate ceramic. Acta Biomater. 2011;7:2007-14

29. Tan YF, Wang G, Fan HS, Wang XL, Lu J, Zhang XD. Expression of core binding factor 1 and osteoblastic markers in C2C12 cells induced by calcium phosphate ceramics in vitro. J Biomed Mater Res Part A 2007:82:152-9.

30. Muller P, Bulnheim U, Diener A, Luthen F, Teller M, Klinkenberg ED, et al. Calcium phosphate surfaces promote osteogenic differentiation of mesenchymal stem cells. J Cell Mol Med. 2008;12:281-91.

31. Yuan H, Yang Z, Li Y, Zhang X, De Bruijn JD, De Groot K. Osteoinduction by calcium phosphate biomaterials. J Mater Sci Mater Med. 1998;9:723-6.

32. Barradas AM, Monticone V, Hulsman M, Danoux C, Fernandes H, Tahmaseb Birgani Z, et al. Molecular mechanisms of biomaterial-driven osteogenic differentiation in human mesenchymal stromal cells. Integr Biol. 2013;5:920-31.

33. Long F. Building strong bones: molecular regulation of the osteoblast lineage. Nat Rev Mol Cell Biol. 2012;13:27-38.

34. Deschaseaux F, Sensébé L, Heymann D. Mechanisms of bone repair and regeneration. Trends Mol Med. 2009;15:417-29.

35. Tang Z, Wang Z, Qing F, Ni Y, Fan Y, Tan Y, et al. Bone morphogenetic protein Smads signaling in mesenchymal stem cells affected by osteoinductive calcium phosphate ceramics. J Biomed Mater Res Part A. 2015;103:1001-10

36. Grundberg E, Brändström H, Lam KCL, Gurd S, Ge B, Harmsen E, et al. Systematic assessment of the human osteoblast transcriptome in resting and induced primary cells. Physiol Genomics. 2008;33:301-11.

37. Ghilardi N, Ziegler S, Wiestner A, Stoffel R, Heim MH, Skoda RC. Defective STAT signaling by the leptin receptor in diabetic mice. PNAS. 1996;93:6231-5.

38. Dumond $\mathrm{H}$, Presle $\mathrm{N}$, Terlain B, Mainard D, Loeuille D, Netter $\mathrm{P}$, et al. Evidence for a key role of leptin in osteoarthritis. Arthritis Rheum. 2003;48:3118-29.

39. Welt CK, Chan JL, Bullen J, Murphy R, Smith P, DePaoli AM, et al. Recombinant human leptin in women with hypothalamic amenorrhea. N Engl J Med. 2004;351:987-97.

40. Baas J, Svaneby D, Jensen TB, Elmengaard B, Bechtold J, Soballe K. Coralline hydroxyapatite granules inferior to morselized allograft around uncemented porous Ti implants: unchanged fixation by addition of concentrated autologous bone marrow aspirate. J Biomed Mater Res A. 2011;99:9-15.

41. Boden SD, Martin Jr GJ, Morone M, Ugbo JL, Titus L, Hutton WC. The use of coralline hydroxyapatite with bone marrow, autogenous bone graft, or osteoinductive bone protein extract for posterolateral lumbar spine fusion. Spine. 1999:24:320-7.

42. Nickoli MS. Ceramic-based bone grafts as a bone grafts extender for lumbar spine arthrodesis: a systematic review. Global Spine J. 2014:4:211-6.

43. Arinzeh TL, Tran T, Mcalary J, Daculsi G. A comparative study of biphasic calcium phosphate ceramics for human mesenchymal stem-cell-induced bone formation. Biomaterials. 2005;26:3631-8.

44. Siggers $\mathrm{K}$, Frei H, Fernlund $\mathrm{G}$, Rossi F. Effect of bone graft substitute on marrow stromal cell proliferation and differentiation. J Biomed Mater Res A. 2010;94:877-85.

45. Dorozhkin SV. Calcium orthophosphate-based bioceramics. Materials. 2013;6:3840-942

46. Draenert M, Draenert A, Draenert K. Osseointegration of hydroxyapatite and remodeling-resorption of tricalciumphosphate ceramics. Microsc Res Tech. 2013;76:370-80

47. Tancred DC, McCormack BA, Carr AJ. A synthetic bone implant macroscopically identical to cancellous bone. Biomaterials. 1998;19:2303-11.

48. Schliephake H, Neukam FW, Klosa D. Influence of pore dimensions on bone ingrowth into porous hydroxylapatite blocks used as bone graft substitutes. A histometric study. Int J Oral Maxillofac Surg. 1991;20:53-8.

49. Vani R, Girija EK, Elayaraja K, Parthiban PS, Kesavamoorthy R, Narayana KS. Hydrothermal synthesis of porous triphasic hydroxyapatite/( $\alpha$ and $\beta$ ) tricalcium phosphate. J Mater Sci Mater Med. 2009;20:S43-8.

50. Yuan $\mathrm{H}$, van Blitterswijk CA, de Groot $\mathrm{K}$, de Bruijn JD. A comparison of bone formation in biphasic calcium phosphate (BCP) and hydroxyapatite (HA) implanted in muscle and bone of dogs at different time periods. J Biomed Mater Res. 2006:78A:139-47.

51. Habibovic P, Yuan H, van der Valk CM, Meijer G, van Blitterswijk CA, de Groot K. 3D microenvironment as essential element for osteoinduction by biomaterials. Biomaterials. 2005;26:3565-75.

52. Liu C, Batliwalla F, Li W, Lee A, Roubenoff R, Beckman E, et al. Genome-wide association scan identifies candidate polymorphisms associated with differential response to anti-TNF treatment in rheumatoid arthritis. Mol Med. 2008;14:575-81.

53. White CD, Brown MD, Sacks DB. IQGAPs in cancer: a family of scaffold proteins underlying tumorigenesis. FEBS Lett. 2009;583:1817-24.

54. Tseng S, Reddi AH, Di Cesare PE. Cartilage oligomeric matrix protein (COMP): a biomarker of arthritis. Biomark Insights. 2009;4:33-44.

\section{Submit your next manuscript to BioMed Central and take full advantage of:}

- Convenient online submission

- Thorough peer review

- No space constraints or color figure charges

- Immediate publication on acceptance

- Inclusion in PubMed, CAS, Scopus and Google Scholar

- Research which is freely available for redistribution 